\title{
Erratum to: Changes in volatiles in carrots inoculated with ACC deaminase-producing bacteria isolated from organic crops
}

\author{
Hiroaki Matsuoka • Yoshinari Ohwaki • \\ Junko Terakado-Tonooka $\cdot$ Fukuyo Tanaka
}

Published online: 30 March 2016

(C) Springer International Publishing Switzerland 2016

\section{Erratum to: Plant and Soil}

DOI: 10.1007/s11104-015-2769-x

The original version of this paper contains errors. In Materials and methods, the accession numbers of the nucleotide sequence are erroneously given as LO075700 to LO075709, and LO075711. The correct numbers are LC075700 to LC075709, and LC075711.

The online version of the original article can be found at $\mathrm{http} / / \mathrm{dx}$. doi.org/10.1007/s11104-015-2769-x.

H. Matsuoka • Y. Ohwaki · J. Terakado-Tonooka •

F. Tanaka $(\bowtie)$

NARO Agricultural Research Center, 3-1-1 Kannondai,

Tsukuba 305-8666, Japan

e-mail: fukuyot@affrc.go.jp

Present Address:

J. Terakado-Tonooka

Saga University, 1 Honjo-machi, Saga 840-8502, Japan 